\title{
Interplay between shape and composition in bimetallic nanoparticles revealed by an efficient optimal-exchange optimization algorithm
}

\author{
Felix Neumann \\ Chair of Theoretical Chemistry and Catalysis Research Center, \\ Technische Universität München, Garching, Germany \\ Johannes Margraf and Karsten Reuter \\ Chair of Theoretical Chemistry and Catalysis Research Center, \\ Technische Universität München, Garching, Germany and \\ Fritz-Haber-Institut der Max-Planck-Gesellschaft, Berlin, Germany \\ Albert Bruix* \\ Chair of Theoretical Chemistry and Catalysis Research Center, \\ Technische Universität München, Garching, Germany and \\ Departament de Ciència de Materials i Química Física and Institut de Química \\ Teòrica i Computacional (IQTCUB), Universitat de Barcelona, Barcelona, Spain
}

\begin{abstract}
Despite the large relevance of bimetallic metal nanoparticles for heterogeneous catalysis, the relation between their shape and elemental composition remains elusive. Here, we investigate this relationship by implementing and applying global optimization methods enhanced with a novel optimal-exchange algorithm. In particular, we determine the lowest energy chemical orderings for $\mathrm{PtAu}$ nanoparticles, revealing that the most stable shape changes from highly symmetric structures for pure particles to distorted and less symmetric shapes for intermediate compositions. The presented method leverages the local atomic contributions to an empirical surrogate energy expression to identify optimal atom exchanges. This also allows us to pinpoint the origin of the stability of distorted shapes, revealing a favorable energy trade-off when over-coordinating $\mathrm{Pt}$ and undercoordinating $\mathrm{Au}$ upon distorting the particle shape.
\end{abstract}

Bimetallic nanoparticles (NP), or nanoalloys, are widely studied due to their applications in optics, magnetism, catalysis, and other energy-related applications [1]. The large number of possible metal combinations offers ample opportunities for adapting to very specific applications and property requirements. For example, the chemical (and resulting catalytic) properties of bimetallic materials can be tailored for activating different reactions by the choice of metals and their relative concentration $[2,3]$. However, determining the structures formed when combining two metals is challenging, even for bulk mixtures crystallizing into fcc-like lattices [4]. Surfaces and NPs involve even more complex spaces of nonequivalent chemical orderings [1], which is why most studies determining the stable structure of large bimetallic NPs have focused on identifying the most stable chemical orderings only for fixed particle shapes, i.e. the most stable homotop [5-7].

Investigating the chemical ordering for fixed shapes provides valuable insights regarding the stability of different surface sites, but the structure and resulting shape of metal NPs are indeed known to depend on their composition. Equilibrium shapes derived from the Wulff construction [8] vary along different monometallic [9] and bimetallic particles [10]. Even more drastic structural transitions involving departures from fcc-like structures can emerge in bimetallic particles when combining weakly miscible metals with a large size-mismatch. In such cases, symmetry-breaking transitions from an icosahedral structure to a decahedral one can occur as a means of releasing strain [11].

Despite the ample evidence pointing to a strong dependence, the interplay between chemical ordering and shape of large ( $>100$ atoms) particles remains unclear and is inaccessible by current computational structure prediction approaches. For example, shapes derived from the Wulff construction [10] assume the preservation of bulk chemical ordering, and the aforementioned single- and fixed- shape approaches are limited by design. To address this, we have developed a novel and efficient global optimization approach exploiting energy decomposition into atomic contributions to evaluate both chemical ordering and shape in bimetallic NPs. We showcase this approach by studying technologically relevant $[12,13]$ $\mathrm{PtAu}$ nanoalloys, for which we demonstrate that intricate shape transitions occur as a function of the relative $\mathrm{Pt}: \mathrm{Au}$ concentration.

Our approach separates the shape and chemical ordering optimization tasks. We first obtain an ensemble of hundreds of single-metal particles with different stable shapes by carrying out a shape sampling and optimization procedure. Subsequently, we search for the most stable chemical ordering for each shape of the ensemble and for each relative concentration of the two metals, providing the relative energies between all shapes as a function of metal concentration. 
Both shape and ordering optimization tasks use surrogate models to evaluate the NP energies at each optimization step. These models rely on a graph partitioning scheme and a topological energy approximation to compute (local) atomic contributions to the energy of single-metal and bimetallic NPs. In addition to providing sufficiently accurate NP total energies, the separation into local contributions allows us to implement a optimalexchange schemes to accelerate established global optimization methods.

This method is formulated for large ( $>100$ atoms) nanoalloys of metals with small size mismatch such as those formed by gold and platinum [14]. Local relaxation effects in such particles are small and involve a minimal loss of crystallinity, which allows constraining the optimization problem to changes in occupation of crystal lattice positions. The energies of bimetallic particles in the resulting space of conformations can be approximated by surrogate models based on e.g. bond-centric parameters [15] or topological descriptors [6]. These approaches significantly reduce the computational cost with respect to first principles approaches commonly used for such systems. The Hamiltonian for the topological descriptor model proposed by Kozlov et al. [6] (analogous to a transverse field Ising model) is expressed as

$$
\mathcal{H}=\sum_{i}^{N} \sigma_{i} E_{\mathrm{CN}_{i}}^{\mathrm{coor}}+\frac{1}{2} \sum_{<i, j>}^{N} E_{\sigma_{i} \sigma_{j}}^{\mathrm{bond}}+E_{0}
$$

where $\sigma_{i}$ is a variable assigned to each of the $\mathrm{N}$ atoms of the NP that takes values $\{0,1\}$ depending on the atomic identity (i.e. platinum or gold in our study). $E_{\mathrm{CN}_{i}}^{\text {coor }}$ corresponds to the contribution to the total energy of atom $i$ with coordination number $\mathrm{CN}_{i}$, and $E_{\sigma_{i} \sigma_{j}}^{\text {bond }}$ is the contribution of a bond formed between atoms $i$ and $j$. The first term thus accounts for how often one of the two metals occupies positions with different coordination numbers, whereas the second accounts for the number of gold-gold, gold-platinum and platinum-platinum bonds of the system. We note that more negative values calculated from eq. 1 correspond to more stable homotops.

To obtain the $E_{\sigma_{i} \sigma_{j}}$ and $E_{\sigma_{i}, \sigma_{j}}$ parameters, we fit eq. 1 to a set of known energies of NPs with different chemical orderings by means of linear regression. The NP energies are calculated by means of the Effective Medium Theory (EMT) approach $[16,17]$ as implemented in the asap calculator of the Atomic Simulation Environment [18]. This allows testing and validating results using large data sets obtained at very low computational cost. Calculations based on Density-Functional Theory (DFT) are also used to validate the most important results obtained in this work and to show the general applicability of our approach to obtain DFT-like accuracy while using a dataefficient strategy. Details regarding the accuracy of the topological energy expression and the DFT calculation parameters are described in the Supporting Information
(Fig. S1).

Global optimization approaches such as Markov-chain Monte Carlo [6] and GAs [5] have been previously used to explore the vast configurational space of possible homotops for given particle shapes and compositions and find the lowest-energy chemical ordering. However, Monte Carlo simulations are often inefficient due to their inability to escape deep basins of the potential energy surface, whereas GAs not complemented with explicit relaxation steps decrease slowly within basins.

To overcome these limitations, we have developed a relaxation and basin-climbing approach that can improve the efficiency of algorithms that globally optimize the chemical ordering in bimetallic NPs. This approach relies on approximations to the local energy of each metal atom derived from eq. 1, which reveal optimal permutations to be carried out by the optimization algorithm. The identification of optimal permutations implemented here is analogous to the deterministic search scheme proposed by Schebarchov and Wales [19], which was itself based on the heuristic approaches for partitioning graphs [20,21].

The search for the most stable chemical ordering is typically carried out by iteratively permuting pairs of atoms such that the energy of the system decreases. Since the number of possible exchange pairs grows as $\mathcal{O}\left(N^{2}\right)$ in the number of atoms of the particle, a random selection of pairs of atoms to be permuted becomes inefficient for large particles. It is more convenient to instead select permutations based on "flip energies" $E_{i}^{\text {flip }}$ [19], which we define as the energy change when replacing the atom type at a given site. We note that the most favorable exchanges correspond to those involving the most negative $E_{i}^{\text {flip }}$ values.

The energy change due to the exchange of two atoms $\mathrm{i}$ and $\mathrm{j}$ can then be approximated by $\Delta E_{i j} \approx E_{i}^{\text {flip }}+$ $E_{j}^{\text {flip }}$, which becomes exact in the case of eq. 1 if two non-neighboring atoms are considered. Obtaining the flip energies is straightforward due to the local nature of the Hamiltonian. Particularly, the local energy of an atom $i$ is only dependent on the number of neighboring $\mathrm{Au}$ atoms $(a)$ and $\mathrm{Pt}$ atoms $(b)$, such that

$$
F_{\sigma_{i}}(a, b)=\sigma_{i} E_{(a+b)}^{\text {coor }}+a E_{\sigma_{i}, 1}^{\text {bond }}+b E_{\sigma_{i}, 0}^{\text {bond }},
$$

where $\sigma_{i}$ is 0 for Pt and 1 for $\mathrm{Au}$. The flip gain for any atom is then expressed as difference in environment energies of the two elements in the same atomic environment:

$$
E_{i}^{\mathrm{flip}}(a, b)=(-1)^{\sigma_{i}}\left[F_{1}(a, b)-F_{0}(a, b)\right] .
$$

Examples of thus calculated flip energies are shown in Fig. 1 for an AuPt NP with both a random compositional ordering and a locally optimal ordered structure. The flip energies readily provide an intuitive evaluation of the stability of local structural motifs. The opposite sign of the flip energies for the two elements indicates that $\mathrm{Pt}$ 


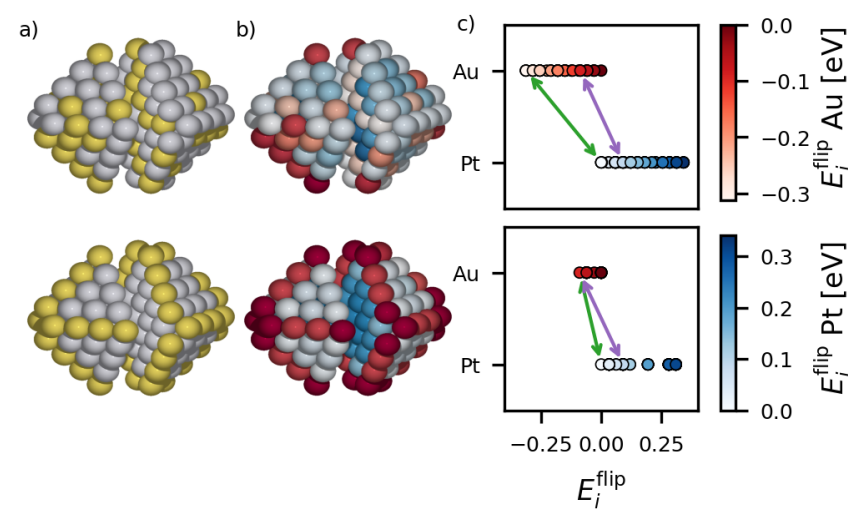

FIG. 1. a) Structure and b) flip energies of a $\mathrm{Au}_{47} \mathrm{Pt}_{93}$ truncated octahedron with random compositional ordering (top) and in a local ordering minimum (bottom). Darker colors indicate more stable atoms. c) Sorted flip energies for the two particles shown, indicating optimal permutations for relaxation (green arrows) and perturbation (purple arrows) steps.

forms stronger bonds than Au. This is also reflected in the optimal atom ordering at this shape, where all $\mathrm{Au}$ atoms occupy undercoordinated edge sites.

Obtaining the $E_{i}^{\mathrm{flip}}(a, b)$ for all atoms of a NP also allows to identify the optimal permutation step, which involves an atom from each element with the respective lowest flip energies. The selection of atom pairs corresponding to the optimal permutation based on sorted flip energies is illustrated in Fig. 1. Consecutive executions of such optimal permutations constitute a deterministic local relaxation of the chemical ordering, reaching a minimum when further energy decrease by exchanging two atoms is no longer possible.

We have implemented the optimal-exchange relaxation algorithm described above within two global optimization frameworks widely used in atomic structure prediction: Basin Hopping (BH) [22] and Genetic Algorithm (GA) approaches.

$\mathrm{BH}$ approaches consist of consecutive perturbation and relaxation steps (see flow chart in Fig. S2 in SI), which allow hopping to different basins and finding the minimum within a basin, respectively. Our implementation of $\mathrm{BH}$ uses the optimal-exchange relaxation for the latter minimization steps. The success of $\mathrm{BH}$ furthermore depends on a judicious choice of perturbation steps, as very destabilizing perturbations are often immediately reverted by the local optimization. [23] An optimal perturbation should therefore allow escaping a basin while keeping the energy of the system low.

Efficient perturbations also rely on flip energies. Specifically, pairs of atoms are selected iteratively, starting from the most stable ones and until the resulting exchange leads to an energy increase. The consecutive execution of such climb-out steps results in a smooth and gradual escape from local minima. The number of energy-increasing permutations executed during a perturbation and the number of perturbation-relaxation cycles are hyper-parameters of the algorithm.

The optimal-exchange relaxation has also been implemented into a GA, such that generated candidates are relaxed to an adjacent local minimum (see details in the SI).

Figure 2 illustrates the performance of the global optimization of the chemical ordering of a truncated octahedral $\mathrm{Au}_{106} \mathrm{Pt}_{209}$ particle using the optimal-exchange $\mathrm{BH}$, the GA with and without optimal-exchange relaxations and simple Monte Carlo (MC) runs. The cumulative success rate for all algorithms has been calculated by running each algorithm 100 times (from different starting points) and evaluating the fraction of runs that have found the (known) global minimum as a function of the number of steps. A step here is defined as the generation and total energy evaluation of one candidate structure.

Without local optimization, the "bare" GA is unable to locate the global minimum, whereas the $\mathrm{MC}$ algorithm requires a very large $\left(>10^{5}\right)$ number of steps. In contrast, algorithms assisted by the optimal-exchange algorithm are able to find the minimum requiring approximately two orders or magnitude fewer steps. We further note that the effect of the optimal-exchange scheme on performance is even more significant when increasing particle size. Such remarkable improvement allows to comfortably perform a large number of global optimizations for different particles on commodity compute architectures. Despite requiring a similar number of steps to find the global minimum, our implementation of the optimalexchange $\mathrm{BH}$ algorithm is faster in CPU time than the GA with optimal exchange relaxation (see Fig. S3 in SI), and we therefore rely on the former to optimize the orderings of ensembles of $\mathrm{Au}_{n} \mathrm{Pt}_{140-n}$ particles with different shape and composition.

Having established the performance of the method for the chemical ordering optimization of particles with a fixed shape, we next turn to exploring ensembles of different shapes. In order to generate a large ensemble of stable single-metal particle shapes, we reuse the $\mathrm{BH}$ algorithm whereby an element ' $\mathrm{X}$ ' is introduced as a placeholder for an empty fcc lattice site. We carry this optimization out for Pt particles of 140 atoms and recalculate the most stable structures for Au. We find more than 200 structures within a $1.0 \mathrm{eV}$ energy range for $\mathrm{Pt}$ ( $1.4 \mathrm{eV}$ for $\mathrm{Au}$ ), and that the truncated octahedron (TO) is the global minimum for both metals. For every shape, a separate training set consisting of 100 randomly ordered structures with evenly spaced compositions is calculated at the EMT level of theory and used for fitting the parameters of our energy model $\left(E_{\mathrm{CN}_{i}}^{\text {coor }}\right.$ for $\mathrm{CN}_{i}=6,7,8,9,12$ and $E_{0,0}^{\text {bond }}, E_{0,1}^{\text {bond }}, E_{1,1}^{\text {bond }}$, and a term accounting for variable composition). We then optimize the chemical ordering for every particle of the 


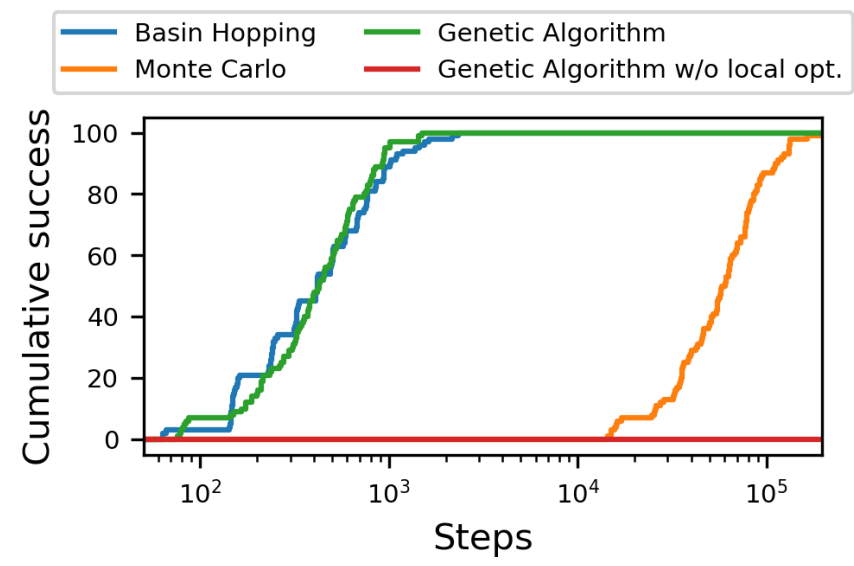

FIG. 2. Cumulative success rates of four different algorithms for optimizing the chemical ordering in $\mathrm{Au}_{106} \mathrm{Pt}_{209}$ particle.

ensemble and for every composition, circumventing the difficulty of optimizing both shape and ordering at the same time. In order to speed up the ordering optimization at each composition, we feed the solution for the particle $\mathrm{Au}_{n} \mathrm{Pt}_{140-n}$ as the starting guess for the next previous composition $\left(\mathrm{Au}_{n+1} \mathrm{Pt}_{139-n}\right)$. This allows performing the $\sim 30 \mathrm{k}$ global optimizations required to find the global minimum for every shape and stoichiometry.
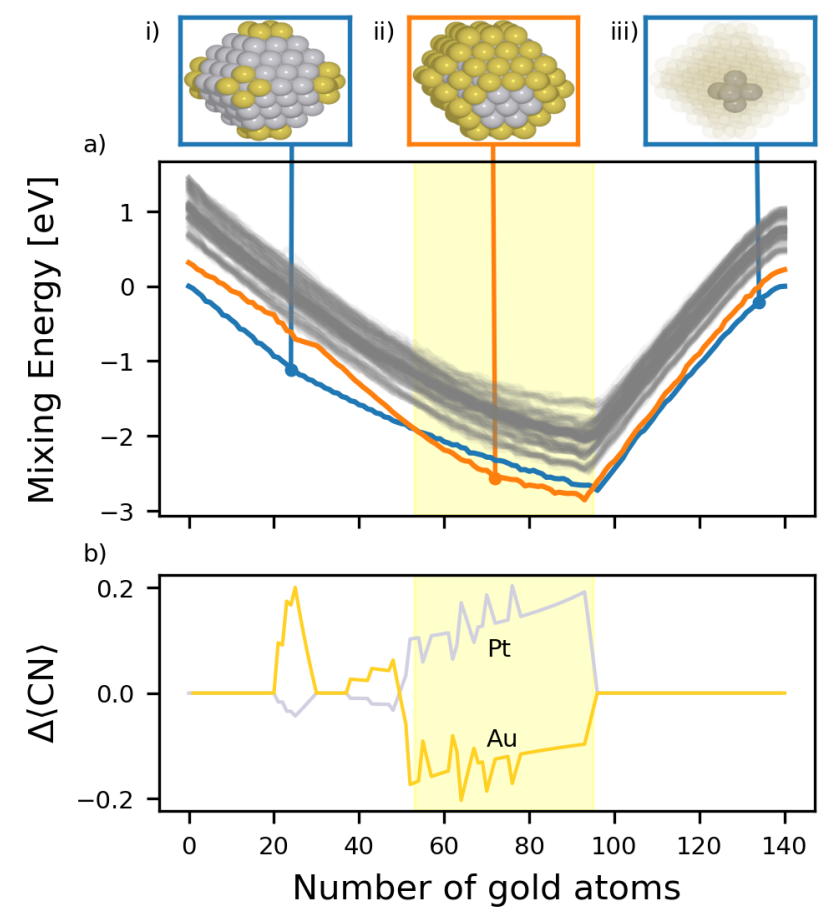

FIG. 3. a) Minimum energy hulls calculated at the EMT level for an ensemble of $160 \mathrm{AuPt}$ particle shapes. b) Difference in the average coordination numbers of $\mathrm{Au}$ and $\mathrm{Pt}$ between the lowest-energy structures of the two shapes.

The lowest-energy chemical orderings found for every shape and composition result in a minimum-energy hull for every shape in the ensemble, illustrating the evolution of the mixing energy within that shape as a function of composition. The mixing energy (also referred to as excess energy) represents the propensity of two metals to form bimetallic particles instead of monometallic ones, and is calculated as

$$
E_{\text {mix }}=E_{\mathrm{Au}_{n} \mathrm{Pt}_{m}}-\frac{n E_{\mathrm{Au}_{n+m}}+m E_{\mathrm{Pt}_{n+m}}}{(n+m)}
$$

where $E_{\mathrm{Au}_{n} \mathrm{Pt}_{m}}$ and $E_{X_{n+m}}$ are the energy of the bimetallic particle and single metal particles (in their most stable truncated octahedral shape), respectively, calculated at the EMT level of theory. Figure 3 illustrates the mixing energies for particles featuring each of the 160 most stable shapes for monometallic Pt. All shapes exhibit a qualitatively similar behavior, with particles of mixed composition being more stable than their monometallic counterparts, $\mathrm{Au}$ atoms preferentially occupying surface positions, and an $E_{\text {mix }}$ minimum in the range between 90 and $100 \mathrm{Au}$ atoms. The structure with lowest $E_{\text {mix }}$ for all shapes corresponds to a perfect coreshell ordering, with a complete Au shell and no bulk-like $\mathrm{Au}$ atoms. The composition at which this ordering is possible depends on the number of surface atoms of each shape, which explains observed variations in the position of the minimum for different minimum-energy hulls.

Two particle shapes are significantly more stable than the rest: the truncated octahedron, which is the minimum energy shape for single metal particles; and a slightly less symmetric shape, which we here refer to as "asymmetrically truncated octahedron" (ATO). Both shapes are shown in the insets of Fig. 3a for different compositions. The minimum-energy hulls of these two structures are highlighted with different colors in Fig. 3a and, remarkably, cross at $\mathrm{Au}_{53} \mathrm{Pt}_{87}$ and $\mathrm{Au}_{97} \mathrm{Pt}_{43}$. The distorted shape (ATO) therefore becomes the most stable one in the yellow-shaded region between these two compositions. Importantly, this shape with composition $\mathrm{Au}_{93} \mathrm{Pt}_{47}$ and a perfect core-shell structure has the overall lowest $E_{\text {mix }}$ among all evaluated shapes, compositions, and chemical orderings, making it the most stable configuration from the millions of evaluated structures.

Changes in relative energies of the different shapes as a function of composition not only affect the lowestenergy shapes, but also those with higher energy. In fact, changes in relative energy are even more pronounced for some highly distorted structures with an increased number of surface atoms and with lower coordination numbers. This indicates that variations in composition not only induce a change in the minimum energy shape, but also makes otherwise very unstable shapes thermally accessible.

The energy approximation in eq. 1 accounts for and quantifies contributions from metal atoms in different co- 
ordination environments. Understanding the transition from the TO to the ATO shape is thus possible by analyzing the varying distribution of coordination numbers for the two metals (see Table SIV). The two shapes have the exact same average coordination number. However, the ATO has more 12-coordinated bulk-like atoms, compensated with an average lower coordination number of surface sites. This distortion in the distribution of coordination numbers is particularly relevant in terms of stability when surface and bulk-like sites are occupied by $\mathrm{Au}$ and $\mathrm{Pt}$ atoms, respectively.

The differences between the two shapes in the average coordination number for $\mathrm{Au}$ and $\mathrm{Pt}$ atoms, $\Delta\langle\mathrm{CN}\rangle=$ $\langle\mathrm{CN}\rangle_{\mathrm{Au}}^{\mathrm{ATO}}-\langle\mathrm{CN}\rangle_{\mathrm{Au}}^{\mathrm{TO}}$ are shown in Fig. 3b and Fig. S6. The most prominent feature in the evolution of $\Delta\langle\mathrm{CN}\rangle$ lies precisely in the yellow-shaded region, where the $\mathrm{Au}$ and Pt in the truncated octahedron are significantly overand under-coordinated, respectively, with respect to the distorted shape. The larger core of the distorted shape therefore allows to over-coordinate $\mathrm{Pt}$ at the expense of under-coordinating Au. For concentrations $n_{\mathrm{Au}}>96$, all surface sites are occupied by $\mathrm{Au}$, the average coordination number of $\mathrm{Au}$ and $\mathrm{Pt}$ are the same for the two shapes, and the truncated octahedron is again the most stable shape. We note the presence of another feature at $\left(20 \leq n_{\mathrm{Au}} \leq 30\right)$. This emerges because Au occupies all (24) corners of the TO first, whereas it is more favorable to occupy some edge positions of the distorted shape before filling all of its corners.

In order to corroborate the composition-induced shape transition with more accurate first-principles calculations, we fit the parameters of eq. 1 to NP energies calculated at the DFT level of theory (see details in SI). In particular, we calculate the energies of NPs with varying composition of both the TO and the ATO. This allows optimizing the chemical ordering of every stoichiometry and generate the corresponding DFT-based minimumenergy hull for each shape, as shown in Fig. S7. Although mixing energies are larger in magnitude for DFT, the minimum-energy hulls are qualitatively similar to the EMT-derived ones. In particular, they also exhibit a shape transition at intermediate compositions (with crossings at $\sim \mathrm{Au}_{40} \mathrm{Pt}_{100}$ and $\sim \mathrm{Au}_{120} \mathrm{Pt}_{20}$ ). The presence of a shape transition for such intermediate compositions was further confirmed by calculating $\mathrm{E}_{\text {mix }}$ values explicitly at the DFT level theory for selected stoichiometries. The resulting $E_{\mathrm{TO}}-E_{\mathrm{ATO}}$ values for $\mathrm{Au}_{n} \mathrm{Pt}_{140-n}$ stoichiometries with $\mathrm{n}=10,90,93,96$, and 130 are 2.08, $-0.82,-1.18,-0.26$, and $0.63 \mathrm{eV}$, respectively. These results confirm both the composition-induced shape transition and that the EMT approach can qualitatively approximate the properties of bimetallic AuPt NPs.

We next address the interplay between shape and composition for particle sizes that cannot form the symmetric and very stable truncated octahedron shape typical of e.g. 79-, 140-, 201-atom particles. Although these magic

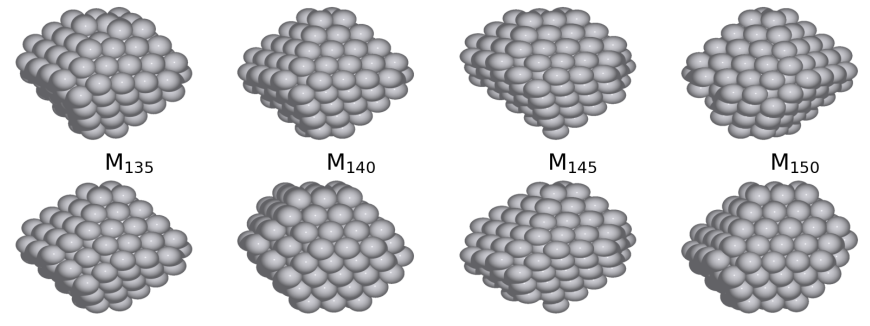

FIG. 4. Most stable (top row) and second most stable (bottom row) Pt NPs of different size.

numbers form particularly stable particles, experimentally prepared metal NPs typically exhibit a rather wide particle size distribution [24]. To generalize the interplay between size and shape to non-magic number particle sizes, we have repeated the minimum-energy hull linescan optimization (fitting eq. 1 to EMT energies) for $\left(\mathrm{Au}_{n} \mathrm{Pt}_{m-n}\right)$ for $\mathrm{m}=135,145$, and 150 . The resulting ensembles of minimum-energy hulls for each of these particle sizes are shown in Figs. S8 to S11, and the two most relevant structures for each size are shown in Fig. 4.

For these non-magic particle sizes, the low-energy structures similarly feature $\mathrm{Au}$ at surface positions. Consequently, $E_{\text {mix }}$ minima also appear at compositions allowing complete $\mathrm{Au}$ shells and $\mathrm{Pt}$ cores. Variations in relative energies with composition are observed for all sizes, leading to clear shape transitions for $\mathrm{Au}_{n} \mathrm{Pt}_{140-n}$ and $\mathrm{Au}_{n} \mathrm{Pt}_{150-n}$. For $\mathrm{Au}_{n} \mathrm{Pt}_{135-n}$ and $\mathrm{Au}_{n} \mathrm{Pt}_{145-n}$, nearly degenerate shapes compete in stability along the entire composition range. In addition, for $\mathrm{Au}_{n} \mathrm{Pt}_{135-n}$ several unstable shapes become nearly as stable as the global minimum for $\mathrm{n} \approx 70-80$. The variations in composition therefore affect the stability ordering for all particle sizes evaluated here, indicating that composition-induced shape changes are general and occur regardless of size for metals with sufficiently different surface energies. A similar example was recently observed on $\mathrm{Ag}$ deposited on Pd surfaces [25], which undergoes a reconstruction where Pd encapsulates Ag.

In conclusion, the shape of bimetallic NPs markedly depends on the composition even for combinations of fcc metals of similar atom size such as $\mathrm{Pt}$ and $\mathrm{Au}$. This behavior emerges in various particle sizes and suggests that fine-tuning the composition can maximize the abundance of desired surface sites. Nevertheless, interaction with adsorbed reactants $[26,27]$ and with a support [28] should be considered for a complete picture of these shape transitions under relevant conditions.

$\mathrm{AB}$ acknowledges the support from the Alexander von Humboldt Foundation, the Generalitat de Catalunya (AGAUR grant 2018BP00190), and the Spanish Ministerio de Ciencia y Universitades (grant MDM-20170767). Computational resources have been provided by the Red Española de Supercomputación (projects QS- 
2020-3-0015, QS-2020-1-0017, and QS-2020-3-0030).

* corresponding author: abruix@ub.edu

[1] R. Ferrando, J. Jellinek, and R. L. Johnston, Chemical Reviews 108, 845 (2008).

[2] M. Andersen, S. V. Levchenko, M. Scheffler, and K. Reuter, ACS Catalysis 9, 2752 (2019).

[3] K. Tran and Z. W. Ulissi, Nature Catalysis 1, 696 (2018).

[4] S. Lysgaard, D. D. Landis, T. Bligaard, and T. Vegge, Topics in Catalysis 57, 33 (2014).

[5] P. C. Jennings, S. Lysgaard, J. S. Hummelshøj, T. Vegge, and T. Bligaard, npj Computational Materials 5, 46 (2019).

[6] S. M. Kozlov, G. Kovács, R. Ferrando, and K. M. Neyman, Chemical Science 6, 3868 (2015).

[7] P. M. Larsen, K. W. Jacobsen, and J. Schiøtz, Physical Review Letters 120, 256101 (2018).

[8] G. Wulff, Zeitschrift für Kristallographie - Crystalline Materials 34, 449 (1901).

[9] R. Tran, Z. Xu, B. Radhakrishnan, D. Winston, W. Sun, K. A. Persson, and S. P. Ong, Scientific Data 3, 160080 (2016).

[10] E. Ringe, R. P. Van Duyne, and L. D. Marks, Nano Letters 11, 3399 (2011).

[11] R. Ferrando, Journal of Physics Condensed Matter 27, 013003 (2015).

[12] C. Xie, Z. Niu, D. Kim, M. Li, and P. Yang, Chemical Reviews 120, 1184 (2020).

[13] J. Suntivich, Z. Xu, C. E. Carlton, J. Kim, B. Han, S. W. Lee, N. Bonnet, N. Marzari, L. F. Allard, H. A. Gasteiger, K. Hamad-Schifferli, and Y. Shao-Horn, Journal of the American Chemical Society 135, 7985 (2013).

[14] P. Janthon, S. M. Kozlov, F. Viñes, J. Limtrakul, and F. Illas, Journal of Chemical Theory and Computation 9, 1631 (2013).
[15] Z. Yan, M. G. Taylor, A. Mascareno, and G. Mpourmpakis, Nano Letters 18, 2696 (2018).

[16] K. W. Jacobsen, P. Stoltze, and J. K. Nørskov, Surface Science 366, 394 (1996).

[17] K. W. Jacobsen, J. K. Norskov, and M. J. Puska, Physical Review B 35, 7423 (1987).

[18] A. Larsen, J. Mortensen, J. Blomqvist, and K. Jacobsen, Journal of Physics: Condensed Matter 29, 273002 (2017).

[19] D. Schebarchov and D. J. Wales, The Journal of Chemical Physics 139, 221101 (2013).

[20] B. W. Kernighan and S. Lin, The Bell System Technical Journal 49, 291 (1970), the Bell System Technical Journal.

[21] C. M. Fiduccia and R. M. Mattheyses, Proceedings - Design Automation Conference, 175 (1982).

[22] D. J. Wales and J. P. Doye, Journal of Physical Chemistry A 101, 5111 (1997).

[23] C. Panosetti, K. Krautgasser, D. Palagin, K. Reuter, and R. J. Maurer, Nano Letters 15, 8044 (2015), pMID: 26444084, https://doi.org/10.1021/acs.nanolett.5b03388.

[24] C. T. Campbell, Accounts of chemical research 46, 1712 (2013).

[25] J. S. Lim, J. Vandermause, M. A. Van Spronsen, A. Musaelian, Y. Xie, L. Sun, C. R. O'Connor, T. Egle, N. Molinari, J. Florian, K. Duanmu, R. J. Madix, P. Sautet, C. M. Friend, and B. Kozinsky, Journal of the American Chemical Society 142, 15907 (2020).

[26] B. C. Han, A. Van Der Ven, G. Ceder, and B. J. Hwang, Physical Review B - Condensed Matter and Materials Physics 72, 1 (2005).

[27] J. R. Kitchin, K. Reuter, and M. Scheffler, Physical Review B - Condensed Matter and Materials Physics 77, 1 (2008), arXiv:0801.1144.

[28] N. J. Divins, I. Angurell, C. Escudero, V. Pérez-Dieste, and J. Llorca, Science 346, 620 (2014). 


\title{
Supplementary information for "Interplay between shape and composition in bimetallic nanoparticles revealed by an efficient optimal-exchange optimization algorithm"
}

\author{
Felix Neumann \\ Chair of Theoretical Chemistry and Catalysis Research Center, \\ Technische Universität München, Garching, Germany \\ Johannes Margraf and Karsten Reuter \\ Chair of Theoretical Chemistry and Catalysis Research Center, \\ Technische Universität München, Garching, Germany and \\ Fritz-Haber-Institut der Max-Planck-Gesellschaft, Berlin, Germany \\ Albert Bruix* \\ Chair of Theoretical Chemistry and Catalysis Research Center, \\ Technische Universität München, Garching, Germany and \\ Departament de Ciència de Materials i Química Física and \\ Institut de Química Teòrica i Computacional (IQTCUB), \\ Universitat de Barcelona, Barcelona, Spain
}




\section{CONTENTS}

This document includes supplementary information and data about:

- Implementation details of the optimization algorithms

- Performance benchmark

- Ensemble creation

- Cross-over discussion

- DFT calculations

- Generalization of composition-dependent shape to different particle sizes

Figure list:

- S1. Accuracy of the topoloigical descriptor model.

- S2. Flow chart of the optimal-exchange algorithm and example of run.

- S3. Runtime Performance of optimal-exchange optimization algorithms.

- S4. Putative minima quality of optimal-exchange optimization algorithms.

- S5. Examples of particles in the shape ensemble.

- S6. Evolution of the coordination number for TO and DS for 140-atom NP.

- S7. DFT-derived convex hull and coordination number differences for two shapes of 140-atom particles.

- S8. EMT-derived convex hull and coordination number differences for different shapes of 135-atom particles.

- S9. EMT-derived convex hull and coordination number differences for different shapes of 140-atom particles.

- S10. EMT-derived convex hull and coordination number differences for different shapes of 145-atom particles. 
- S11. EMT-derived convex hull and coordination number differences for different shapes of 150-atom particles.

Table list:

- SI: optimal exchange BH parameters

- SII: Parameters used for the GA optimizations

- SIII: Parameters used for the shape optimization

- SIV: Abundance of sites with different coordination numbers in the most stable shapes found for the 140-atom particle.

\section{IMPLEMENTATION DETAILS OF THE OPTIMIZATION ALGORITHMS}

The exact implementation details and choice of hyperparameters can significantly affect the performance of any optimization algorithm. Very often this choice follows a trialand-error procedure which is hard to justify by underlying principles. Nevertheless do we hope that our parameters and implementations provide a good starting point for future investigations of different systems. All algorithms have been implemented in Python on top of the Atomic Simulation Environment (ASE) package [1] in our own nanoparticle library NPL. The repository, including code and tutorials, is freely available on Github: https://github.com/Themistoflix/NPL

\section{Greedy exchange optimization / Basin Hopping}

The parameters for the perturbation strength and number local relaxations/perturbations are given in the following table:

\section{GA with and without local optimization}

The two GA implementations are conceptually identical except for inclusion of the local optimization procedure directly after the creation of a new offspring particle. We have opted for a pool-based approach where a newly created offspring is immediately added 


$\begin{array}{lccc}\text { Parameter } & 79 \text { atoms } & 140 \text { atoms } & 314 \\ \text { Perturbation Strength } & 10 & 20 & 30 \\ \text { No. of relaxations } & 5 & 20 & 60\end{array}$

TABLE I. Parameters used for the Basin Hopping. The perturbation strength denotes how many exchanges are made during the careful climbout phase. In total, No. of relaxation times the particle is locally relaxed and perturbed again.

to the population. For offspring creation we implemented two operators, the Cut-andSplice operator, and an exchange operator, which would randomly exchange atoms of two species. Multiple exchanges are possible with the with the probability of $n$ exchanges being $p(n)=2^{-n}$. Probabilities for the application were 0.4 for the cut-and-splice operator and 0.6 for the exchange operator, respectively. As fitness function we used $f_{i}=\exp \left(-\alpha \rho_{i}\right)$ with $\rho_{i}$ being the the normalized energy and chose particles for procreation by a probability directly proportional to to their fitness. To maintain the diversity of the population particles with a duplicate energy were filtered out immediately after creation. Especially when including the local optimization procedure this was necessary as a multitude of particles will result in the exact same local minimum. We note that each step is more demanding for the GA, where the calculated fitness of each candidate also involves estimating the structural diversity of the population.

Determining convergence was achieved via counting the generations (i.e. number of produced candidates) which did not improve the minimal energy of the population and finishing after a certain number of unsuccessful generations had passed. Particles which were filtered out due to their similarity to already existing particles would not be counted as unsuccessful generations until this has happened a predefined number of times in a row. Exact parameters values for population size, non-improving generations before termination and maximum number of particles that can be rejected due to non uniqueness are shown for both the GA with and without local optimzation in table II:

\section{Monte Carlo}

In addition to the GA without local optimization we turned to the Metropolis algorithm for a further unbiased method. Best results were achieved using $\beta=150 \frac{1}{\mathrm{eV}} \approx 77 \mathrm{~K}$. The tem- 


\begin{tabular}{|c|c|c|c|}
\hline \multirow{2}{*}{$\begin{array}{l}\text { Parameter } \\
\text { with local optimization }\end{array}$} & \multicolumn{3}{|c|}{79 atoms 140 atoms 314 atoms } \\
\hline & & & \\
\hline Population size & 5 & 10 & 15 \\
\hline Termination after generation & 5 & 10 & 15 \\
\hline Max. iterations & 5 & 10 & 10 \\
\hline \multicolumn{4}{|l|}{ without local optimization } \\
\hline Population size & 15 & 25 & 35 \\
\hline Termination after generation & 400 & 800 & 1200 \\
\hline Max. iterations & 5 & 10 & 10 \\
\hline
\end{tabular}

TABLE II. Parameters used for the GA with and without local optimization.

perature was held constant during the whole process. Again we allowed multiple exchanges to mitigate the risk of getting trapped in local minima by following the same procedure as was applied in the exchange operator of the genetic algorithm.

A convergence criterion based on the total number of possible exchanges has been introduces by Kozlov et al. [2] which we apply: After $2 N_{\mathrm{Pt}} N_{\mathrm{Au}}$ exchanges without improvement the search terminates with a probability of having tried every possible single pair exchange with a probability of $63 \%$.

\section{Energy model}

Obtaining the parameters for the topological descriptors was achieved via linear regression. The training sets consisted of 20 randomly created structures in the case of fixed composition 100 randomly created structures with evenly spaced concentrations for the convex hull optimization. In all cases we obtain the feature vectors for the non-relaxed structures and fit to the energies of the relaxed structures. We highlight here that this approach can describe the energy of AuPt particles with mean absolute errors $<3 \mathrm{meV} /$ atom. . 


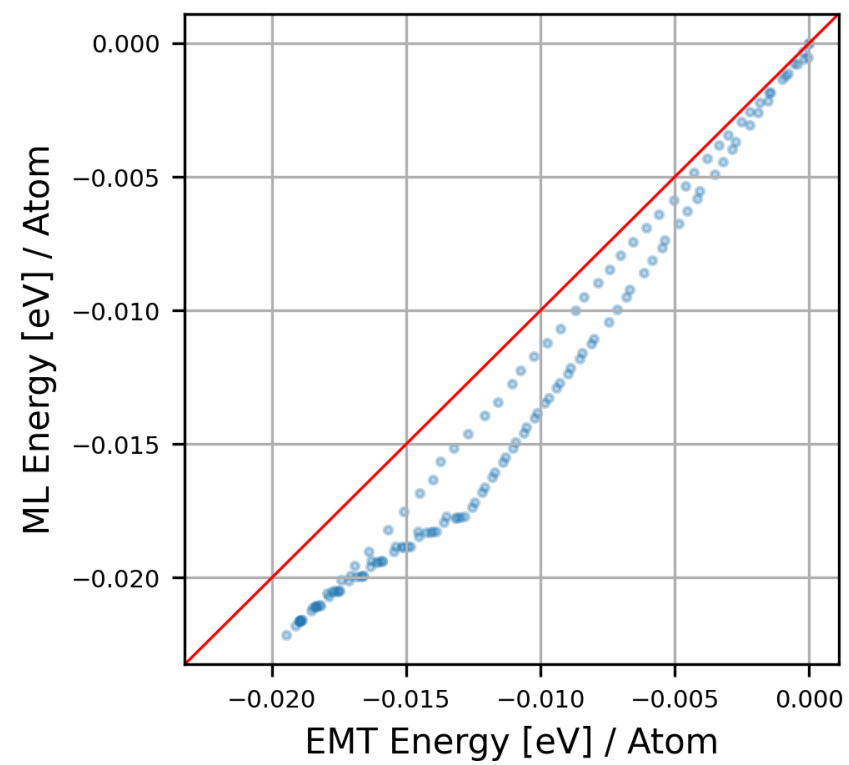

FIG. 1. Parity plot between mixing energies for the $A u_{140-N} \mathrm{Pt}_{\mathrm{N}}$ system calculated at the EMT level and those calculated using the surrogate model. 


\section{BENCHMARK DETAILS}

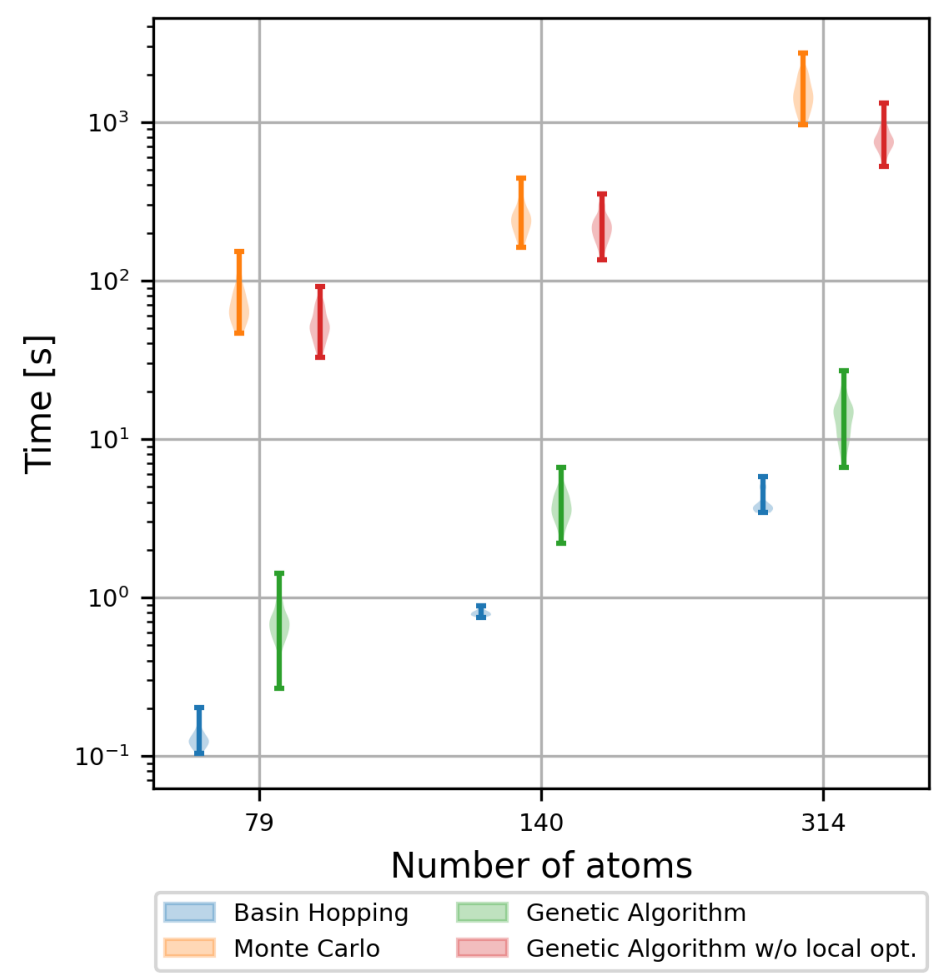

FIG. 2. Run-time comparison of the different algorithms using a single core of a commodity desktop computer. We show this to illustrate relative run-times of different algorithms rather than the absolute numbers (which are highly sensitive to architecture and quickly outdated). 
a
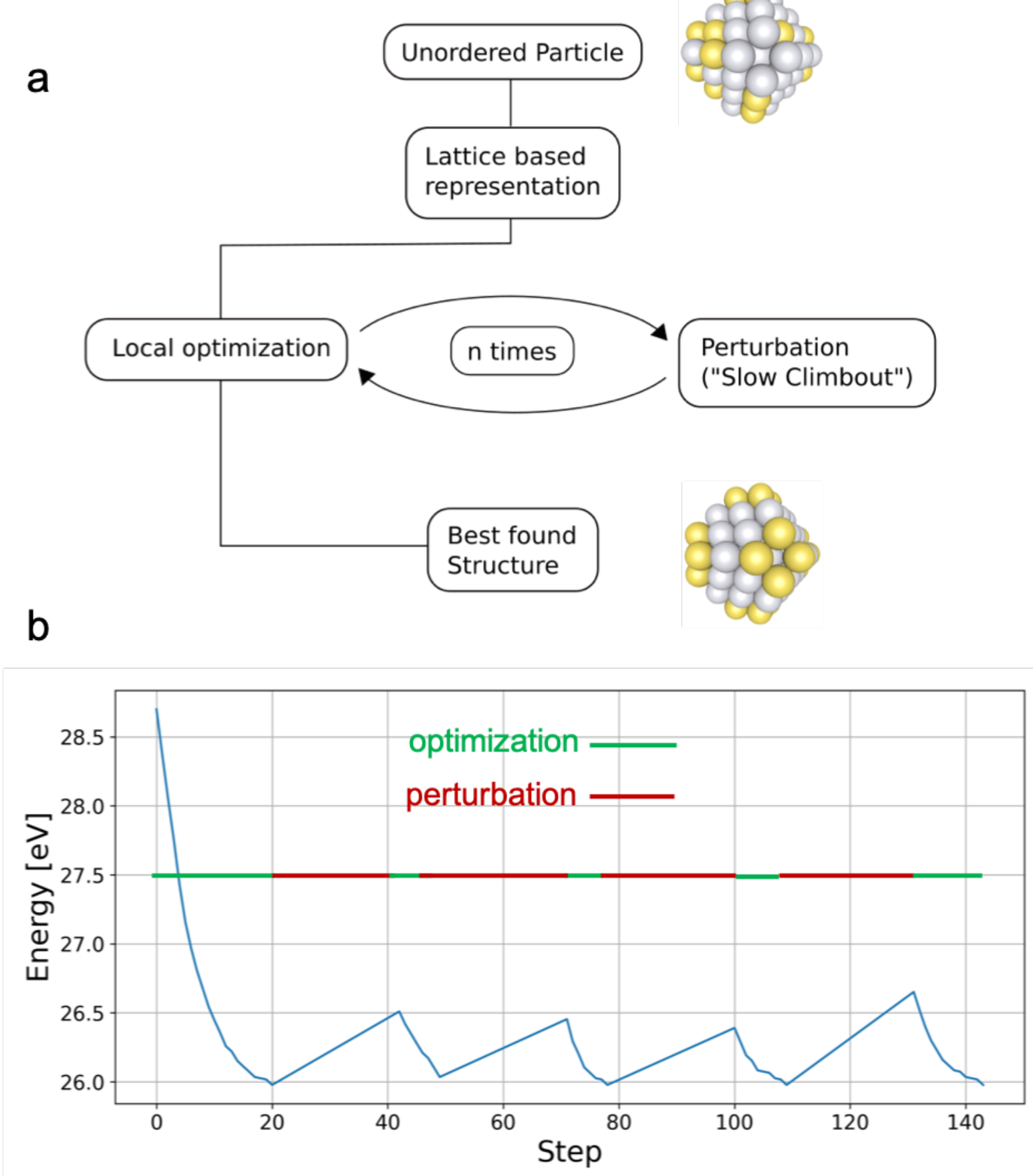

FIG. 3. (a) Flow chart of the optimal-exchange BH algorithm and (b) energy evolution of an exemplary run illustrating the optimization (green) and perturbation (red) steps. 


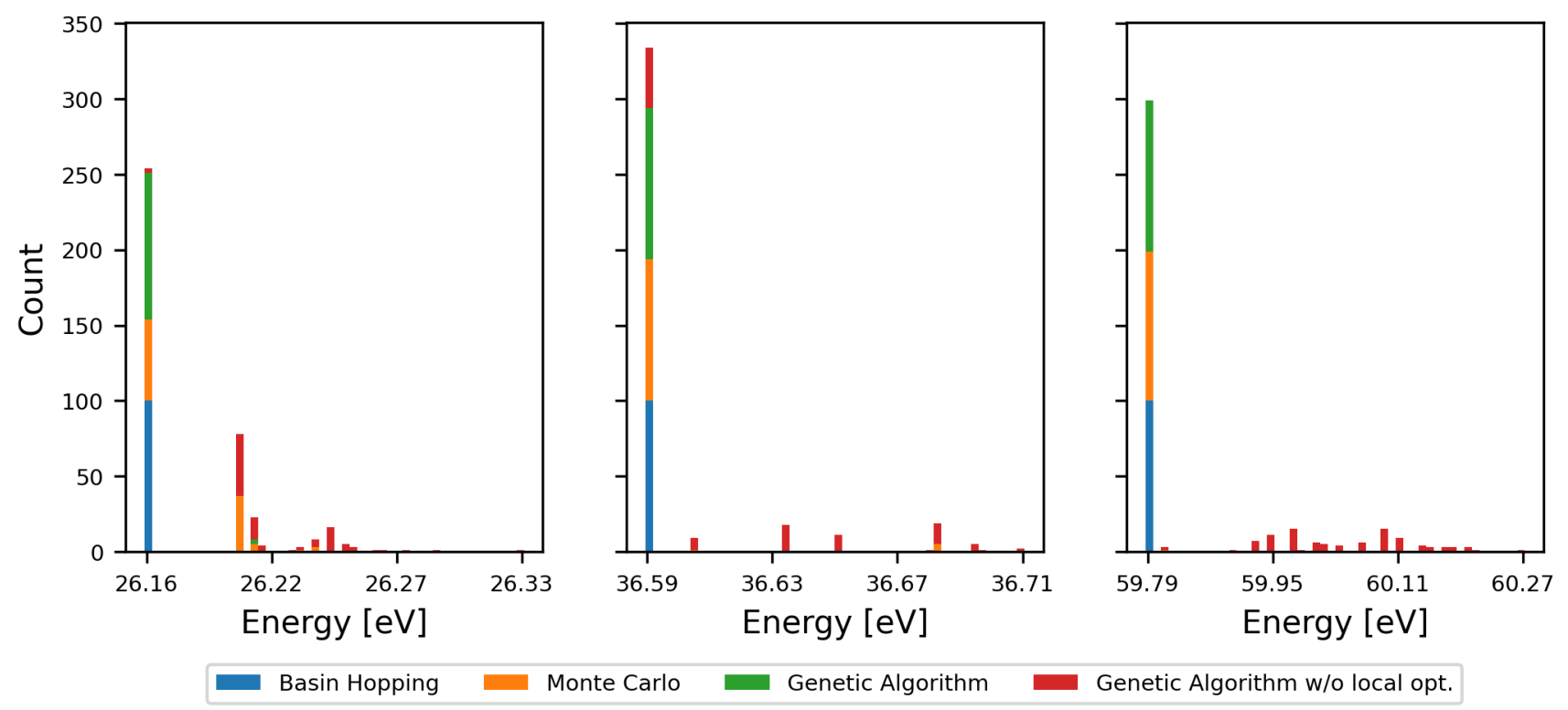

FIG. 4. Distribution of the energies of every algorithm's best solutions after 100 runs. 


\section{ENSEMBLE CREATION}

Obtaining an ensemble of differently shaped but equally sized particles was realised using the $\mathrm{BH}$ algorithm in combination with a purpose-tailored training set. By introducíng an element ' $\mathrm{X}$ ' we are able to map the task of rearranging atoms on a fixed fcc lattice (i.e. shape optmization) to the chemical ordering problem that our method was designed for to solve. However, the problem arises that random orderings then resemble by no means physically meaningful structures, which in turn leads to extremely large displacements of atoms during relaxation and thus renders the use of the topological descriptors impossible. By restricting the relaxation process to one step we circumvent this hurdle as the nominal energies are not of interest in this case. The second challenge that emerges is the fact that the distribution of coordination numbers of a specific particle shifts dramatically during the optimization process, from on average very low to medium/high as a more and more compact structures is formed. Our final training set has to account for this in order to be applicable. By combining three methods for generating porous, semi-compact and compact particles we tried to resemble the different stages of the algorithm and distributions of coordination numbers. The methods were i) random ordering ii) randomly removing surface atoms from compact particles and iii) greedily maximizing the coordination number to create compact particles. Each method contributed 20 particles to our final training set resulting in the parameters shown in table III for the energy model. Across the different sizes $(135,140,145$ and 150 atoms) this set of parameters was reused.

The optimization process was repeated several times for every size, during which we captured all local minima and added them to the ensemble. Recalculating the obtained candidates with EMT allowed us then to identify the 100 (160 for 140 atoms) most stable shapes which we then used as starting point for further investigation. It should be noted, that in case of a monometallic particle

only suitable for smaller shapes as energy model insensitive to higher Miller indices In the case of an monometallic particle the model degenerates to a solely coordination dependent model. 

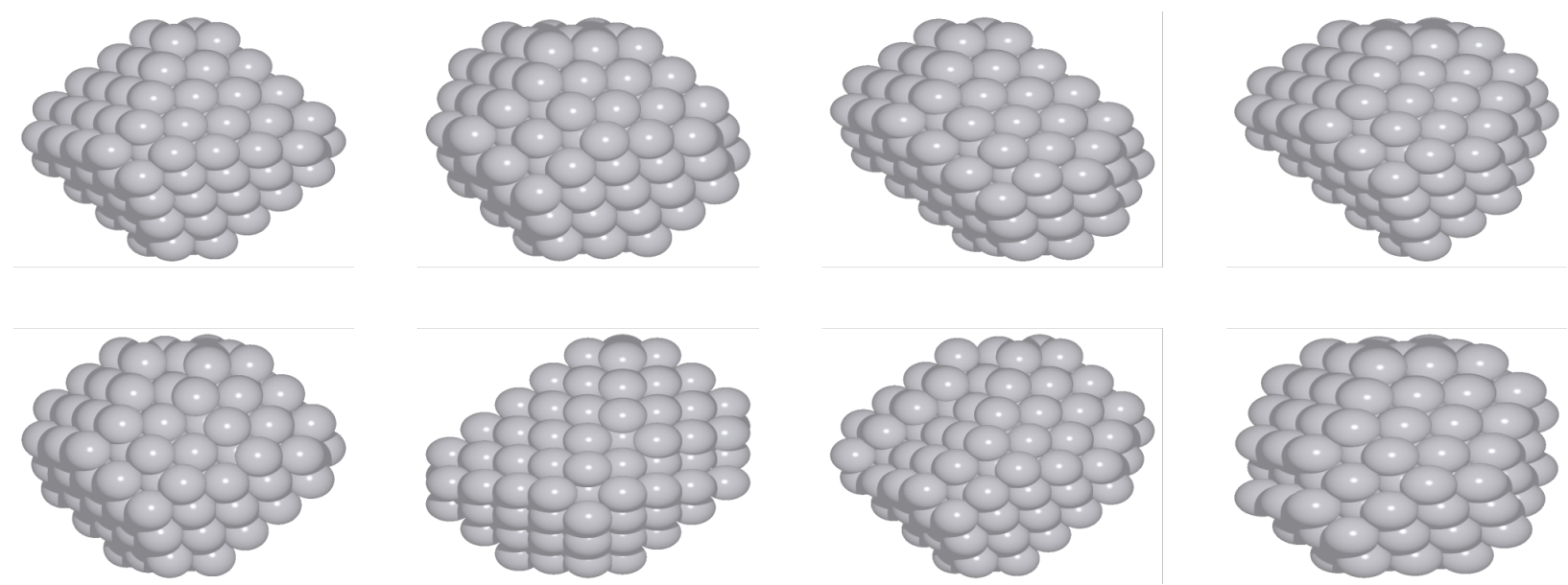

FIG. 5. Particles in the ensemble for 140 atoms, left to right, top to bottom, Shape: 0, 1, 2, 3, 10, $60,110,150$

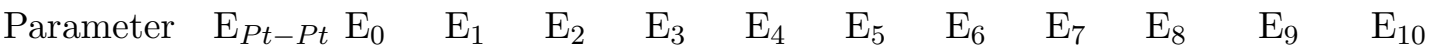
$\mathrm{E}_{11} \quad \mathrm{E}_{12}$

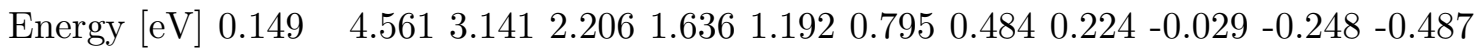
$-0.681 \quad-0.879$
}

TABLE III. Coefficients of the energy model used for the creation of the ensemble of shapes (Pt only). The index denotes the coordination number. 


\section{CROSSOVER DISCUSSION}

\begin{tabular}{lll} 
Descriptor & \multicolumn{2}{l}{ Octahedron \# Distorted Shape \# } \\
Total bonds & 636 & 636 \\
6-fold Pt atoms & 24 & 24 \\
7-fold Pt atoms & 24 & 27 \\
8-fold Pt atoms & 0 & 3 \\
9-fold Pt atoms & 48 & 39 \\
12-fold Pt atoms & 44 & 47
\end{tabular}

TABLE IV. Occurences of features in the octahedron and the distorted particle

Here we also need to include a table with the coordination numbers of the atoms in the two minima of the 140-atom particle.

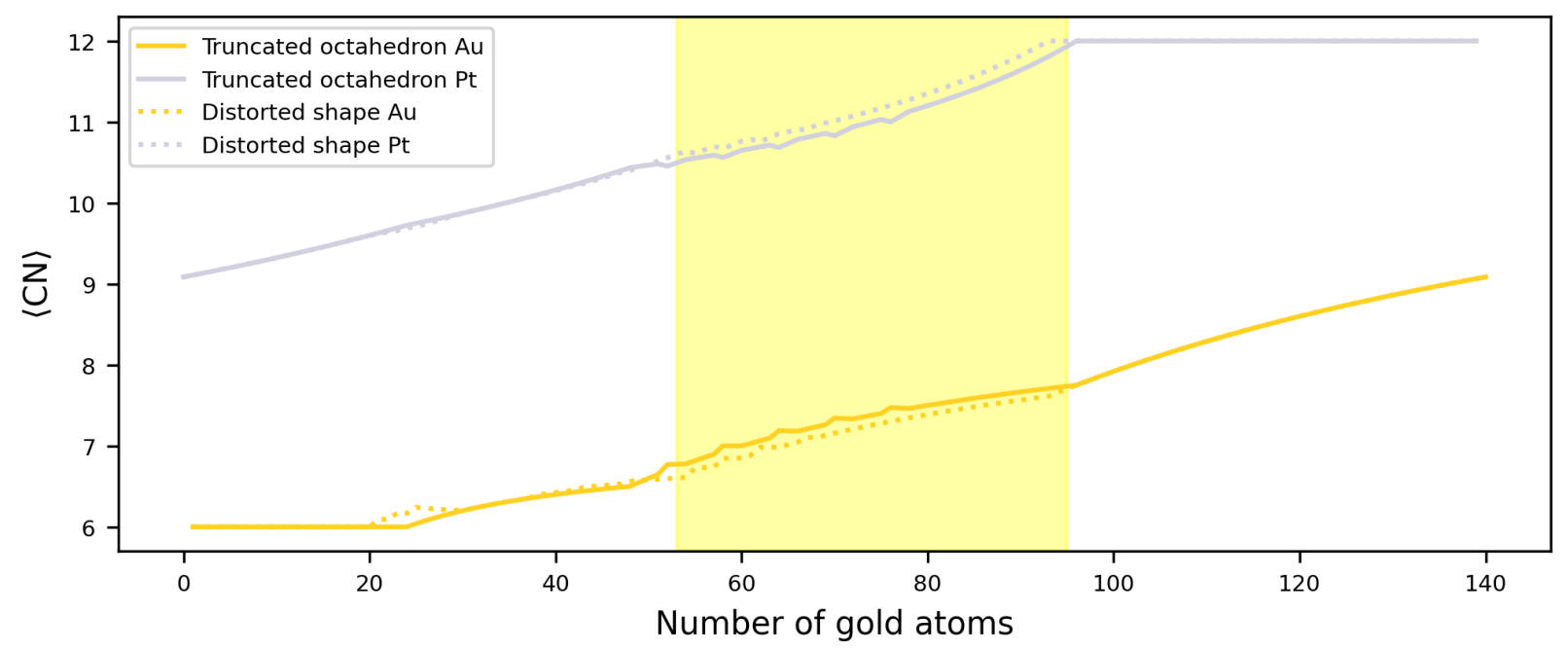

FIG. 6. Evolution with composition of the average coordination of gold and platinum for the TO and distorted shapes of 140-atom PtAu nanoparticles.) 


\section{DENSITY FUNCTIONAL THEORY CALCULATIONS}

\section{Computational details}

All Density Functional Theory (DFT) calculations were carried out with the periodic Quantum Espresso code [3], using ultra-soft pseudopotentials and the van der Waalscorrected BEEF-vdW exchange correlation functional [4]. The Brillouin zone was sampled at the Gamma point only, and cut-offs of 500 and $5000 \mathrm{eV}$ were used for the orbitals and charge density, respectively. The electronic structure was self-consistently calculated until energy differences between consecutive steps were smaller than $5 \mathrm{E}-5 \mathrm{eV}$, and all nanoparticle structures were relaxed until forces for all atoms were smaller than $0.05 \mathrm{eV} / \AA$. In order to avoid interactions between NPs in neighboring unit cells, vacuum was added to the supercell ensuring particle-particle distances $<8 \AA$.

\section{DFT-derived convex hull}

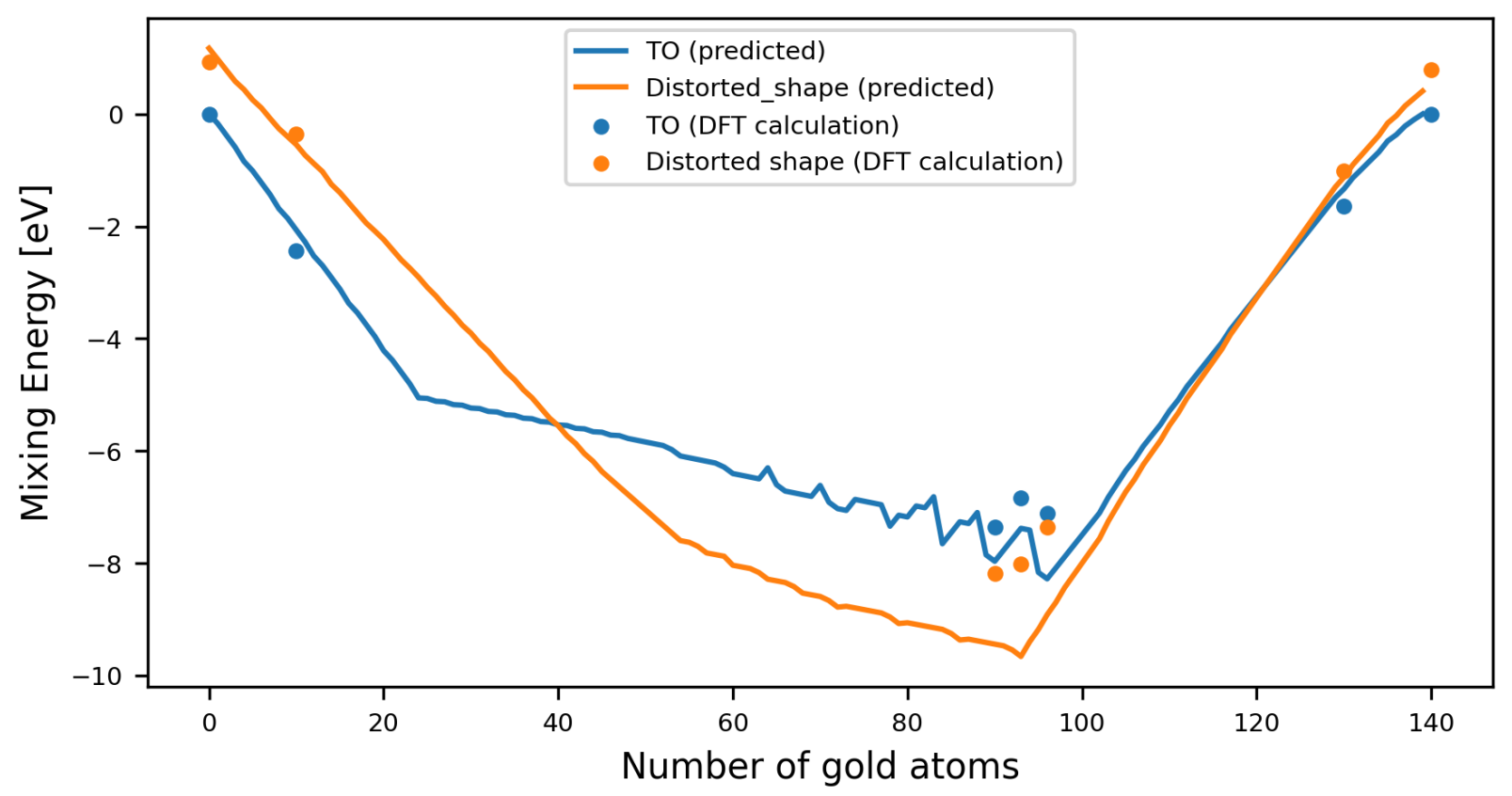

FIG. 7. DFT-derived mixing energy evolution with composition for different shapes of 140-atom particles) 


\section{GENERALIZATION OF COMPOSITION-DEPENDENT SHAPE TO DIFFERENT PARTICLE SIZES}

Convex hulls for different shapes of 135, 140, 145, and 150-atom nanoparticles.

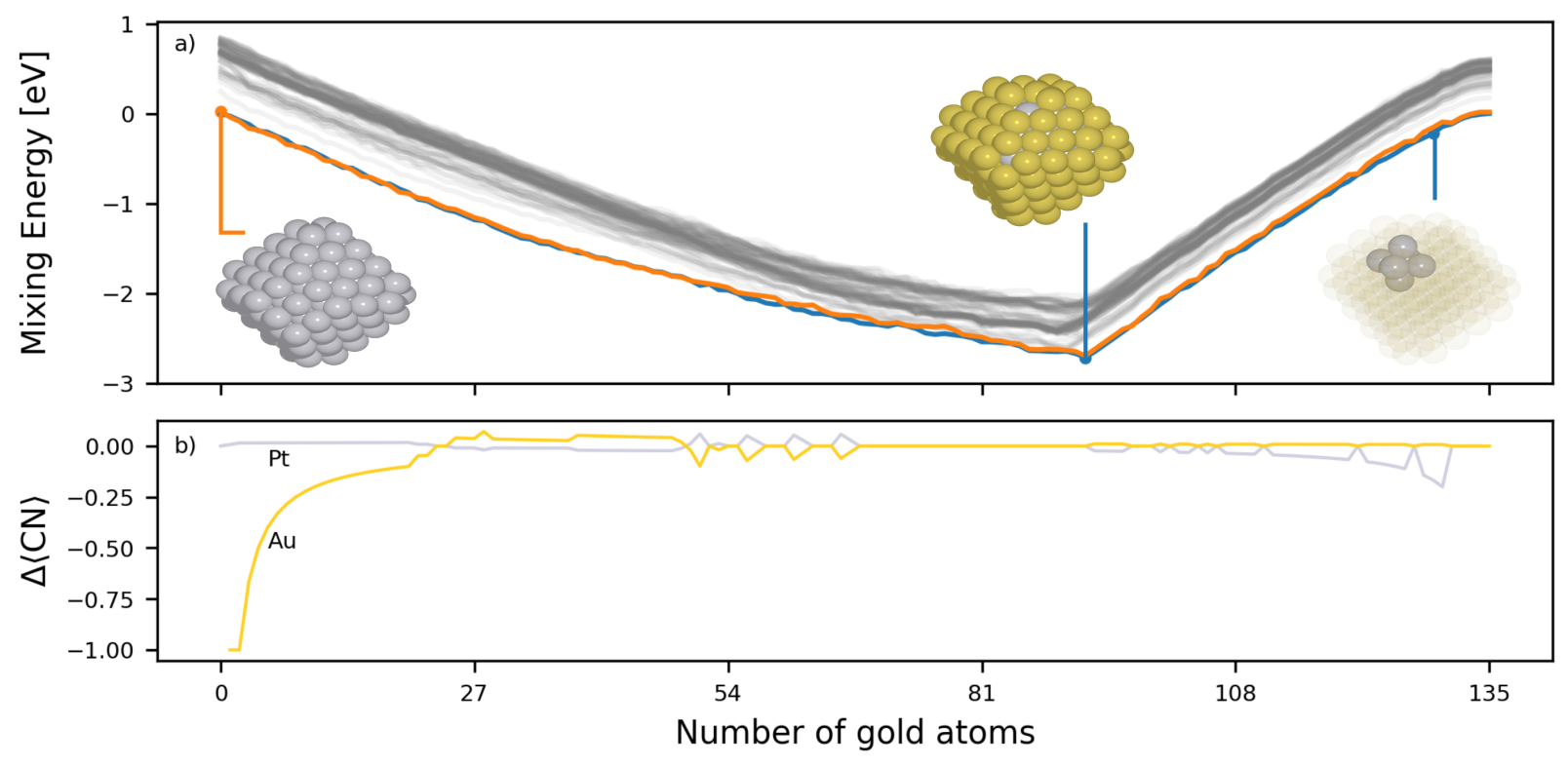

FIG. 8. Mixing energy evolution with composition for different shapes of 135-atom particles .)
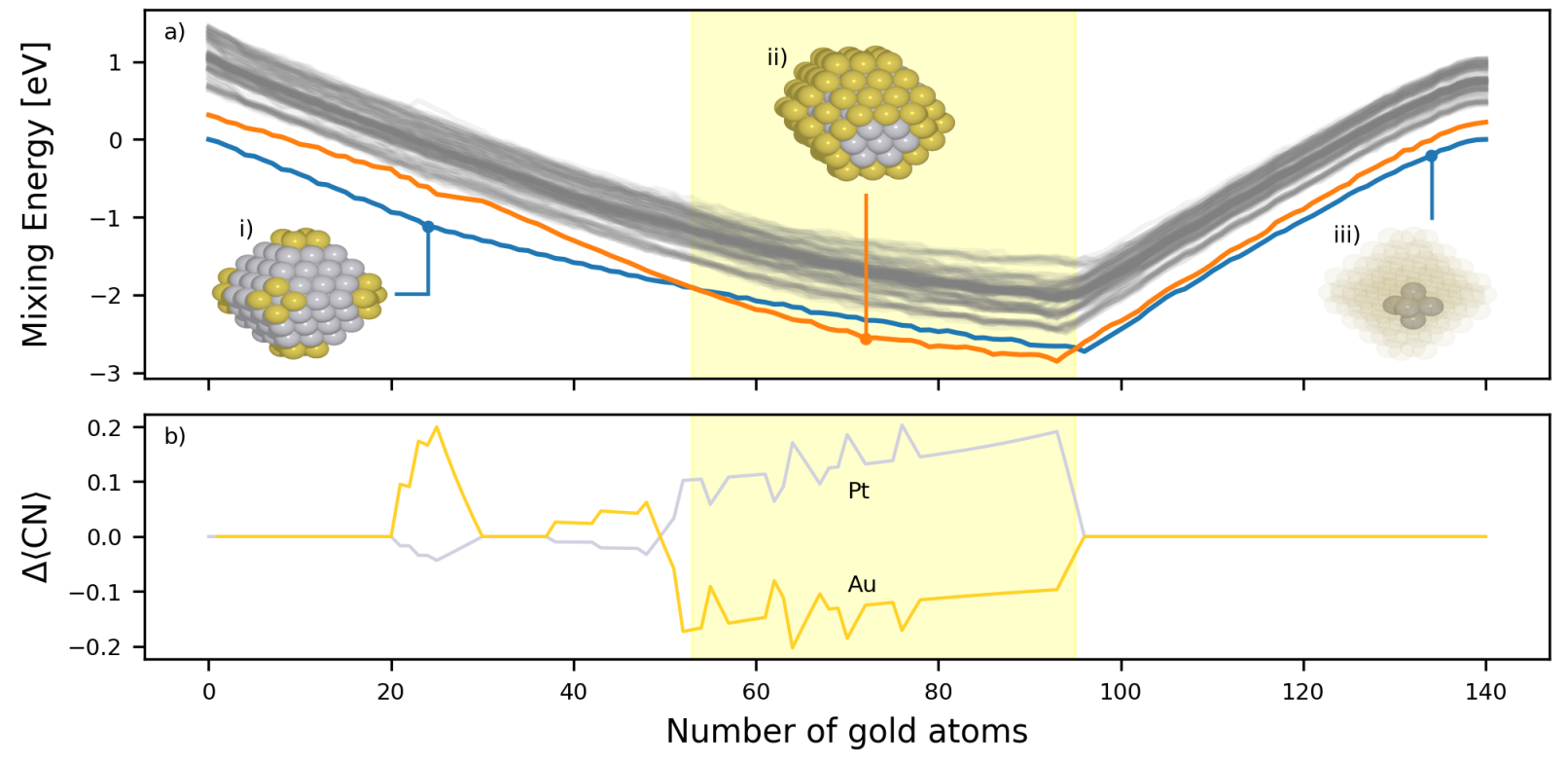

FIG. 9. Mixing energy evolution with composition for different shapes of 140-atom particles) 

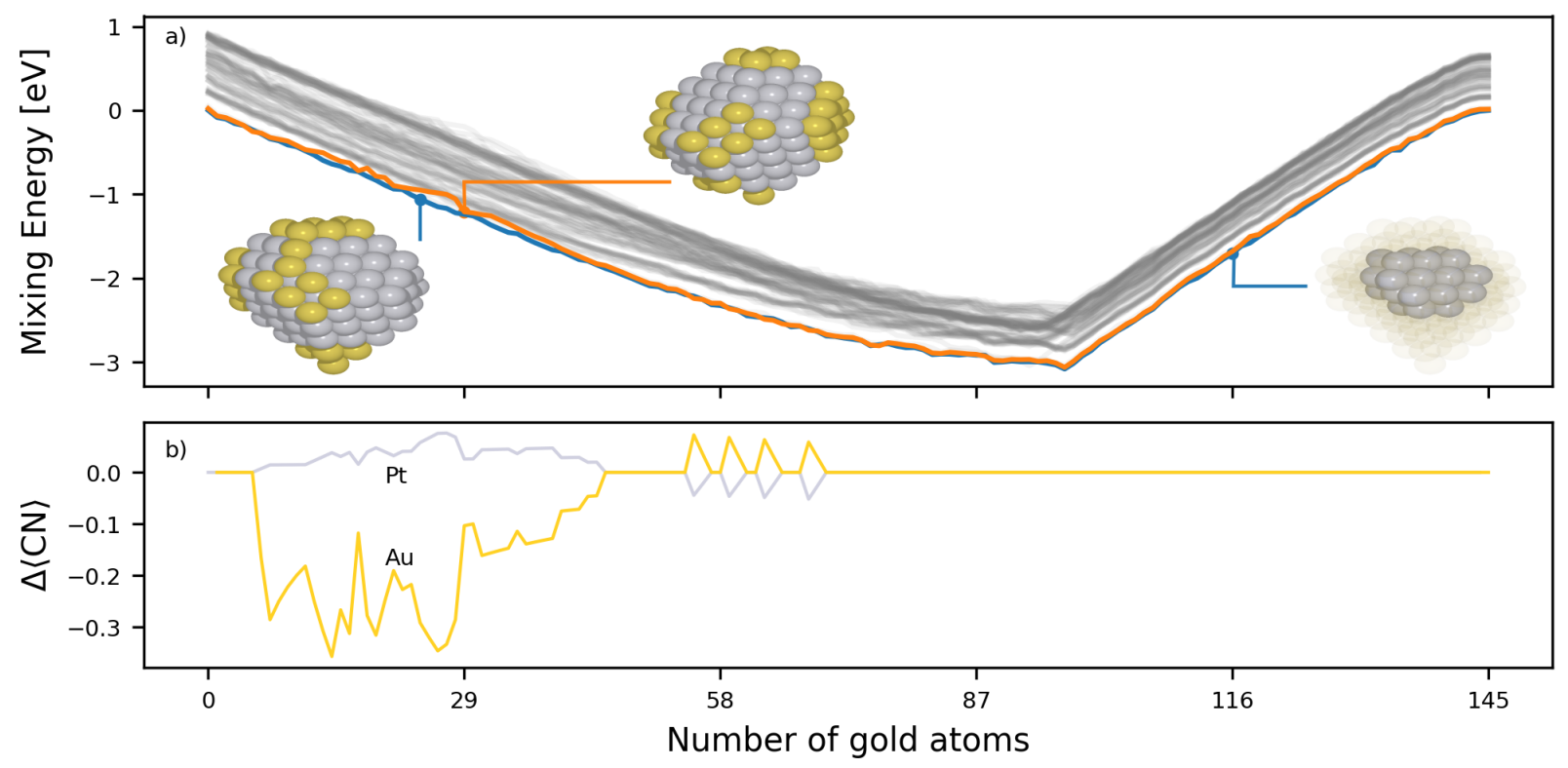

FIG. 10. Mixing energy evolution with composition for different shapes of 145-atom particles)
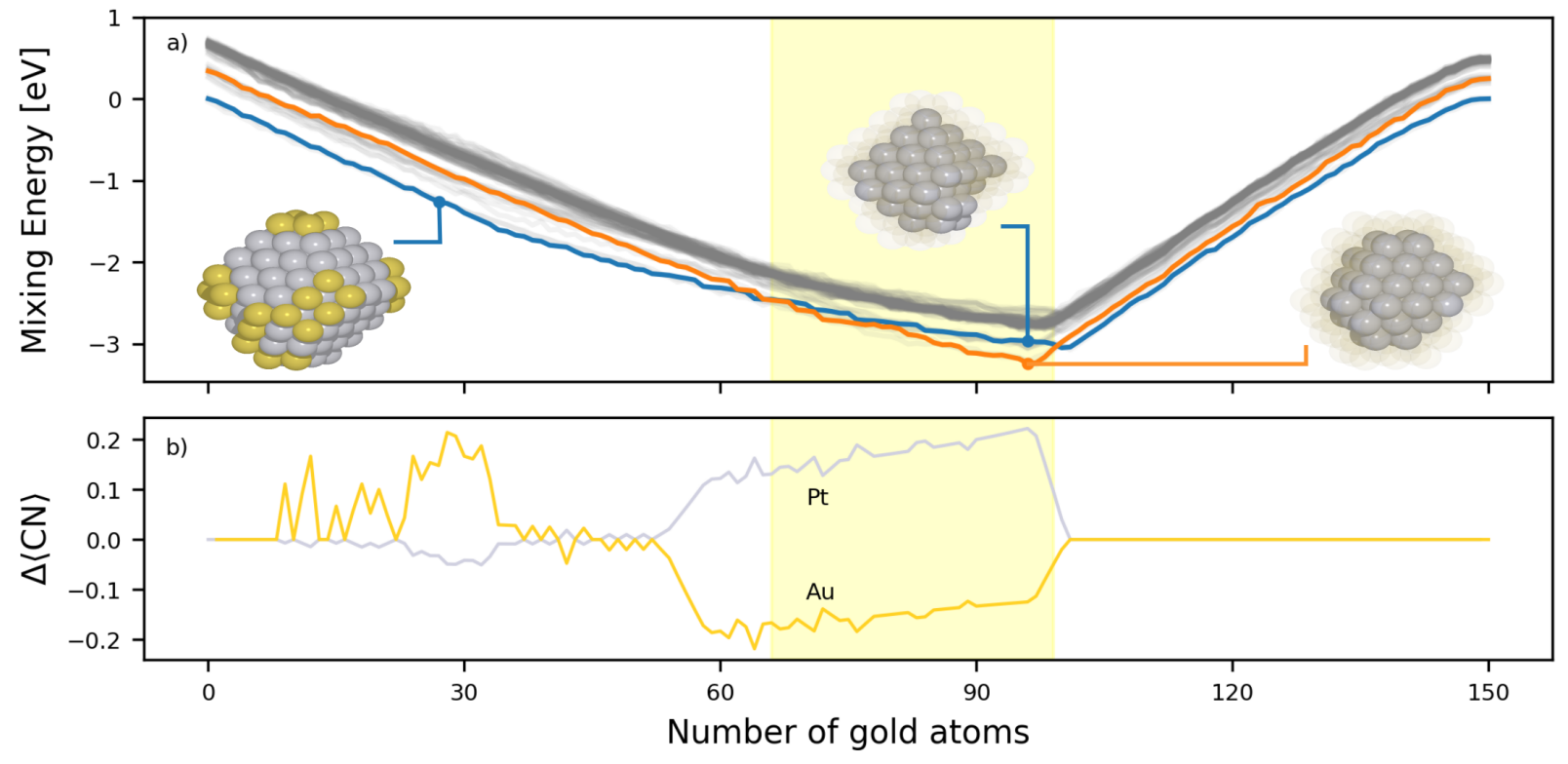

FIG. 11. Mixing energy evolution with composition for different shapes of 150-atom particles)

* corresponding author: abruix@ub.edu 
[1] A. Larsen, J. Mortensen, J. Blomqvist, and K. Jacobsen, Journal of Physics: Condensed Matter 29, 273002 (2017).

[2] S. M. Kozlov, G. Kovács, R. Ferrando, and K. M. Neyman, Chemical Science 6, 3868 (2015).

[3] P. Giannozzi, S. Baroni, N. Bonini, M. Calandra, R. Car, C. Cavazzoni, D. Ceresoli, G. L. Chiarotti, M. Cococcioni, I. Dabo, A. Dal Corso, S. De Gironcoli, S. Fabris, G. Fratesi, R. Gebauer, U. Gerstmann, C. Gougoussis, A. Kokalj, M. Lazzeri, L. Martin-Samos, N. Marzari, F. Mauri, R. Mazzarello, S. Paolini, A. Pasquarello, L. Paulatto, C. Sbraccia, S. Scandolo, G. Sclauzero, A. P. Seitsonen, A. Smogunov, P. Umari, and R. M. Wentzcovitch, Journal of Physics Condensed Matter 21 (2009), 10.1088/0953-8984/21/39/395502, arXiv:0906.2569.

[4] J. Wellendorff, K. T. Lundgaard, A. Møgelhøj, V. Petzold, D. D. Landis, J. K. Nørskov, T. Bligaard, and K. W. Jacobsen, Phys. Rev. B 85, 235149 (2012). 